\title{
Maximal mass of uniformly rotating homogeneous stars in Einsteinian gravity
}

\begin{abstract}
K. Schöbel and M. Ansorg
Theoretisch-Physikalisches Institut, Friedrich-Schiller-Universität Jena, Max Wien Platz 1, 07743 Jena, Germany

Received 6 February 2003 / Accepted 22 April 2003

Abstract. Using a multi domain spectral method, we investigate systematically the general-relativistic model for axisymmetric uniformly rotating, homogeneous fluid bodies generalizing the analytically known Maclaurin and Schwarzschild solutions. Apart from the curves associated with these solutions and a further curve of configurations that rotate at the mass shedding limit, two more curves are found to border the corresponding two parameter set of solutions. One of them is a Newtonian lens shaped sequence bifurcating from the Maclaurin spheroid sequence, while the other one corresponds to highly relativistic bodies with an infinite central pressure. The properties of the configuration for which both the gravitational and the baryonic masses, moreover angular velocity, angular momentum as well as polar red shift obtain their maximal values are discussed in detail. In particular, by comparison with the static Schwarzschild solution, we obtain an increase of $34.25 \%$ in the gravitational mass. Moreover, we provide exemplarily a discussion of angular velocity and gravitational mass on the entire solution class.
\end{abstract}

Key words. stars: rotation - gravitation - relativity - methods: numerical

\section{Introduction}

Various numerical methods have been developed to investigate relativistic rotating models for extraordinarily compact astrophysical objects like neutron stars (Wilson 1972; Bonazzola \& Schneider 1974; Friedman et al. 1986, 1989; Komatsu et al. 1989a, 1989b; Lattimer et al. 1990; Neugebauer \& Herold 1992; Herold \& Neugebauer 1992; Bonazzola et al. 1993, 1998; Eriguchi et al. 1994; Stergioulas \& Friedman 1995; Nozawa et al. 1998). For reviews see Friedman (1998) and Stergioulas (1998).

Although at present more realistic equations of state are explored, we consider in this paper only homogeneous and uniformly rotating star models. This is interesting and important for subsequent computations, because the Newtonian and the static cases are well understood, in particular the analytically known Maclaurin and Schwarzschild solutions. These models were first studied by Butterworth \& Ipser (1975, 1976), who found that, in addition to the analytic solutions, they are bounded by a sequence of configurations rotating at the mass shedding limit ${ }^{1}$. The investigation of such limiting configurations is instructive since certain physical quantities reach maximal values there. With regard to the astronomical observations and a reliable identification of black holes, one is most interested in an upper bound on the gravitational mass and

Send offprint requests to: $\mathrm{K}$. Schöbel,

e-mail: K. Schoebel@tpi .uni-jena.de

${ }^{1}$ Due to uniform rotation, a shedding of matter sets in when centrifugal forces balance gravity at the equator. Then a cusp at the star's equatorial rim appears. especially on how it is affected by rotation. Butterworth \& Ipser for example estimated a $30 \%$ increase for fixed central pressure owing to rotation.

In the present paper we completed their studies, finding two further limiting curves, in particular a sequence of stars with infinite central pressure and a sequence of Newtonian lens-shaped configurations that bifurcates from the Maclaurin spheroids before ending in a mass shedding limit (Bardeen 1971; Ansorg et al. 2003b). All five limiting curves were found to circumscribe entirely the general relativistic solution for homogeneous star models that are continuously joined to the static Schwarzschild solution - hereafter called the "generalized Schwarzschild solution" 2. This was done using the recently developed AKM method (Ansorg et al. 2002, 2003c), which allows one to solve the Einstein equations to high accuracy even for critical configurations. In particular we are able to determine to high precision the extreme configuration possessing both a mass shed and infinite central pressure. At this point several physical quantities reach their global maxima and we provide explicit values for these.

In what follows we use units in which the speed of light and Newton's constant of gravitation assume the value 1 .

\footnotetext{
2 This name (and not for example "generalized Maclaurin solution") was chosen since every possible Schwarzschild solution is contained within this class of solutions. In contrast, only those Maclaurin spheroids with axis ratios above 0.17126 are contained in this class (see Sect. 4).
} 


\section{Metric tensor and field equations}

The line element of a stationary, axisymmetric and asymptotically flat spacetime describing a uniformly rotating perfect fluid body can be cast into the form

$$
\mathrm{d} s^{2}=\mathrm{e}^{-2 U}\left[\mathrm{e}^{2 k}\left(\mathrm{~d} \rho^{2}+\mathrm{d} \zeta^{2}\right)+W^{2} \mathrm{~d} \varphi^{2}\right]-\mathrm{e}^{2 U}(a \mathrm{~d} \varphi+\mathrm{d} t)^{2}
$$

The corresponding Lewis-Papapetrou coordinates $(\rho, \zeta, \varphi, t)$ are uniquely defined if we require continuity of the metric coefficients and their first derivatives at the body's surface. In a comoving frame, for which the metric assumes the same form with metric functions $U^{\prime}, k^{\prime}, W^{\prime}$ and $a^{\prime}$, the relativistic Euler equation can easily be integrated to determine the pressure $p$. For constant mass-energy density $\mu$ this results in

$p=\mu\left(\mathrm{e}^{V_{0}-U^{\prime}}-1\right)$

where $V_{0}$ is the constant surface value of $U^{\prime}$.

Taking into account asymptotic flatness, boundary and transition conditions at the surface and regularity along the rotational axis $(\rho=0)$, the interior and exterior field equations form a complete set of equations to be solved, which is done by applying the AKM method. For a comprehensive discussion of this multi domain spectral method see Ansorg et al. (2003c).

\section{Known static and Newtonian limits}

\subsection{Schwarzschild solution}

Solving Einstein's equation for a static homogeneous star yields the famous Schwarzschild metric which reads in the above coordinates

$\mathrm{e}^{U}=\frac{1-M /(2 r)}{1+M /(2 r)} \quad \mathrm{e}^{k}=1-\left(\frac{M}{2 r}\right)^{2} \quad W=\mathrm{e}^{k} \rho \quad a=0$

for the exterior $\left(r \equiv \sqrt{\rho^{2}+\zeta^{2}} \geq R\right)$ and

$\mathrm{e}^{U^{\prime}}=\frac{1}{2}\left[3 \frac{1-M /(2 R)}{1+M /(2 R)}-\frac{1-M r^{2} /\left(2 R^{3}\right)}{1+M r^{2} /\left(2 R^{3}\right)}\right]$

$\mathrm{e}^{k^{\prime}}=\frac{[1+M /(2 R)]^{3}}{1+M r^{2} /\left(2 R^{3}\right)} \mathrm{e}^{U^{\prime}} \quad W^{\prime}=\mathrm{e}^{k^{\prime}} \rho \quad a^{\prime}=0$

for the interior region $(0 \leq r \leq R)$. Here $M$ is the gravitational mass and $R$ denotes the coordinate radius given implicitly through

$$
M=\mu \frac{4 \pi}{3} R^{3}\left(1+\frac{M}{2 R}\right)^{6} .
$$

For any physically relevant solution the pressure (1) has to remain finite, which is fulfilled for $R>M$. This imposes an upper bound on the mass, namely

$$
M<\frac{4}{9 \sqrt{3 \pi \mu}}=0.14477 \ldots \frac{1}{\sqrt{\mu}}
$$

\subsection{Maclaurin spheroids}

In Newtonian gravity the problem of self gravitating rotating ideal fluids requires solving the Poisson equation for the body's gravitational field while satisfying the Euler-Lagrange equation governing its motion as an ideal fluid. This leads to a free boundary problem. A particular solution for uniform rotation and constant mass density $\mu$ can be found by assuming the surface to be a spheroid. One obtains the so called Maclaurin spheroids, parametrized here by focal distance $\rho_{0}$ and the ratio $r_{\mathrm{p}} / r_{\mathrm{e}}$ between polar radius $r_{\mathrm{p}}$ and equatorial radius $r_{\mathrm{e}}$.

Having computed the gravitational field, the EulerLagrange equation is seen to be satisfied for a squared angular velocity

$\Omega^{2}=2 \pi \mu \xi\left[\left(3 \xi^{2}+1\right) \operatorname{arccot} \xi-3 \xi\right], \quad \xi \equiv\left[\frac{r_{\mathrm{e}}^{2}}{r_{\mathrm{p}}^{2}}-1\right]^{-\frac{1}{2}}$

(bottom solid curve in Fig. 1). This relation holds independent of $\rho_{0}$.

Note that Maclaurin spheroids exist for every $r_{\mathrm{p}} / r_{\mathrm{e}} \in[0,1]$ and $\rho_{0} \in[0, \infty[$, thus comprising a two parameter solution with arbitrary mass for fixed $\mu$.

\subsection{First Newtonian lens sequence}

On the Maclaurin curve, an infinite series of points corresponding to axisymmetric secular instabilities occurs, beginning at $r_{\mathrm{p}} / r_{\mathrm{e}}=0.17126$, and accumulating in the Maclaurin disk limit $r_{\mathrm{p}} / r_{\mathrm{e}} \rightarrow 0$ (Chandrasekhar 1967; Bardeen 1971). They are bifurcation points of further Newtonian sequences and correspond to singular post-Newtonian corrections (see Petroff 2003). The first one of these sequences is comprised of two segments that depart from the first bifurcation point. One segment proceeds towards the Dyson rings (Dyson 1892, 1893; Wong 1974; Kowalewsky 1885; Poincaré 1885a, 1885b, 1885c; Eriguchi \& Sugimoto 1981) whereas the other one ends in a mass shedding limit (Bardeen 1971; Ansorg et al. 2003b). The latter we will refer to as the "first Newtonian lens sequence" motivated by the shape of the corresponding bodies.

\section{Generalized Schwarzschild solution}

For a systematic investigation of the general case the choice of parameters is not restricted by the numerical approach. Nevertheless it is convenient to take non-ambiguous parameters that are restricted to a compact interval (here $[0,1])$ in such a way that the endpoints represent limiting configurations. Corresponding to the limits found, we selected the following magnitudes:

- Mass shed parameter (as defined in Ansorg et al. 2003c)

$$
\beta \equiv-\left.\frac{r_{\mathrm{e}}^{2}}{r_{\mathrm{p}}^{2}} \frac{\mathrm{d}\left(\zeta_{\mathrm{s}}^{2}\right)}{\mathrm{d}\left(\rho^{2}\right)}\right|_{\rho=r_{\mathrm{e}}}=-\frac{r_{\mathrm{e}}}{r_{\mathrm{p}}^{2}} \lim _{\rho \rightarrow r_{\mathrm{e}}} \zeta_{\mathrm{s}} \frac{\mathrm{d} \zeta_{\mathrm{s}}}{\mathrm{d} \rho},
$$

where $\zeta_{\mathrm{s}}(\rho)$ is the function describing the surface shape. Maclaurin and Schwarzschild bodies are characterized by $\beta=1$ and the mass shed limit is fixed by $\beta=0$. 


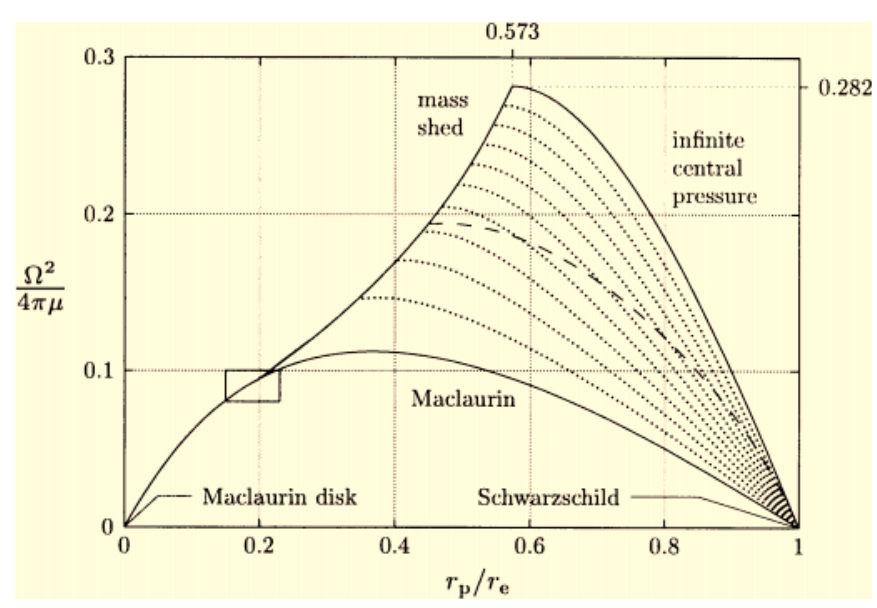

Fig. 1. Squared angular velocity on the generalized Schwarzschild solution. Dotted lines indicate curves of constant central pressure (equally spaced in $\tilde{p}_{\mathrm{c}}=p_{\mathrm{c}} /\left(\mu+p_{\mathrm{c}}\right), p_{\mathrm{c}}$ being the central pressure and $\mu$ the constant mass density). Ergo-toroids appear for configurations situated above the dashed line.

- $\tilde{p}_{\mathrm{c}} \equiv p_{\mathrm{c}} /\left(\mu+p_{\mathrm{c}}\right)$, where $p_{\mathrm{c}}$ is the central pressure. Thus $\tilde{p}_{\mathrm{c}}=0$ stands for the Newtonian limit where mass and hence pressure vanish and $\tilde{p}_{\mathrm{c}}=1$ for the limiting configurations with infinite central pressure.

Additionally we will use the ratio $r_{\mathrm{p}} / r_{\mathrm{e}}$ of polar to equatorial coordinate radius that is 1 only for Schwarzschild solutions.

Our numerical analysis revealed that the generalized Schwarzschild solution is entirely bounded by the following five limiting curves (joined in the order listed):

- the sequence of Schwarzschild solutions $\left(r_{\mathrm{p}} / r_{\mathrm{e}}=1, \beta=1, \tilde{p}_{\mathrm{c}} \in[0,1]\right)$;

- the Maclaurin sequence from the sphere to the first axisymmetric bifurcation point $\left(r_{\mathrm{p}} / r_{\mathrm{e}} \in[0.171,1], \beta=1, \tilde{p}_{\mathrm{c}}=0\right)$;

- the first Newtonian lens sequence $\left(r_{\mathrm{p}} / r_{\mathrm{e}} \in[0.171,0.192], \beta \in[0,1], \tilde{p}_{\mathrm{c}}=0\right)$;

- a sequence of configurations rotating at the mass shedding $\operatorname{limit}\left(r_{\mathrm{p}} / r_{\mathrm{e}} \in[0.192,0.573], \beta=0, \tilde{p}_{\mathrm{c}} \in[0,1]\right)$;

- a sequence of configurations with infinite central pressure $\left(r_{\mathrm{p}} / r_{\mathrm{e}} \in[0.573,1], \beta \in[0,1], \tilde{p}_{\mathrm{c}}=1\right)$.

This makes it possible to determine maximal values of all interesting physical quantities (see Sect. 5). Moreover, we can now state that on the generalized Schwarzschild solution no quasistationary transition to a Kerr black hole is possible (in contrast to what was found for the relativistic Dyson rings by Ansorg et al. 2003a) and that the surface remains convex in $\rho$ $\zeta$-coordinates.

Figure 1 depicts the (squared) angular velocity versus the radius ratio $r_{\mathrm{p}} / r_{\mathrm{e}}$. This is the completion of results of Butterworth \& Ipser $(1975,1976)^{3}$. It shows that the configuration with maximal angular velocity rotates at the mass shedding limit and possesses infinite central pressure. The magnification in Fig. 2 demonstrates that the mass shedding curve does not

\footnotetext{
${ }^{3}$ Note that in contrast to our work they used proper radial distances and kept the baryonic mass constant.
}

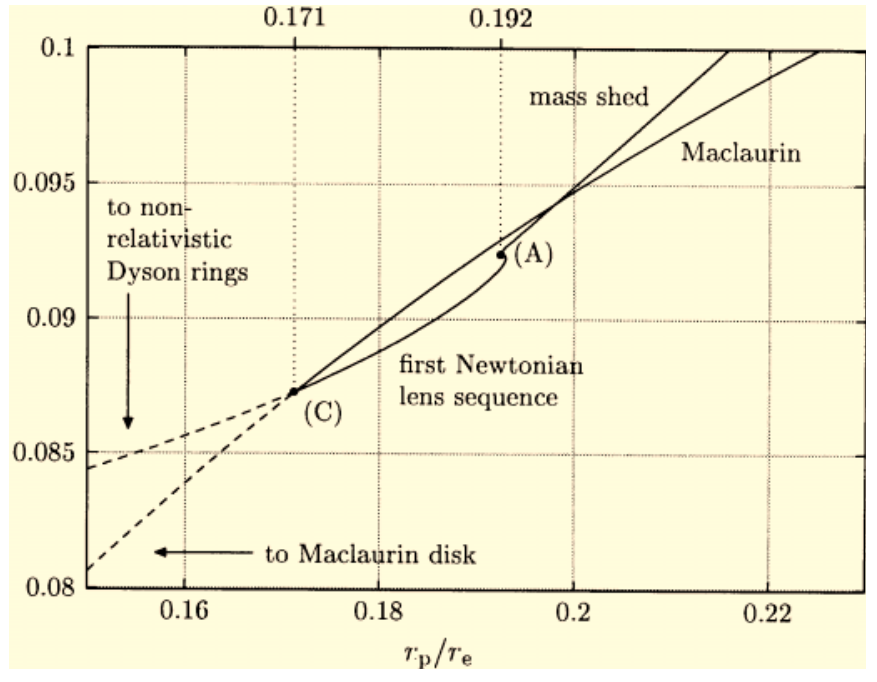

Fig. 2. Magnification of Fig. 1 in the vicinity of the first axisymmetric bifurcation point $(\mathrm{C})$. Values for the first Newtonian lens sequence were taken from Ansorg et al. (2003b). Points (A) and (C) correspond to the cross sections thus labelled in Fig. 6 ibid.

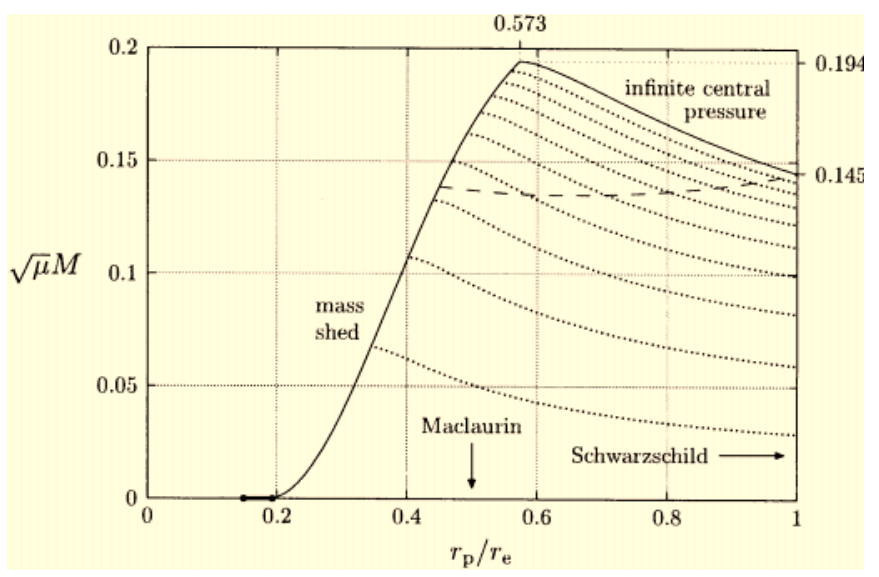

Fig. 3. Gravitational mass on the generalized Schwarzschild solution. The small interval on the $r_{\mathrm{p}} / r_{\mathrm{e}}$-axis, emphasized by a thick line, represents the first Newtonian lens sequence. Dotted lines indicate curves of constant central pressure (equally spaced in $\tilde{p}_{\mathrm{c}}$, see Fig. 1). Ergo-toroids appear for configurations situated above the dashed line.

terminate at the first axisymmetric bifurcation point (C), as was conjectured by Butterworth and Ipser. Instead it is linked to the Maclaurin spheroids at this point via the first Newtonian lens sequence.

The evolution of the gravitational mass for fixed central pressure is seen in Fig. 3 and reveals a $34.25 \%$ increase in the maximal mass with respect to the static case. The global maximum is again found for the same configuration as for angular velocity. In both figures we included the line above which ergoregions appear. Interestingly, this line corresponds roughly to one of constant mass.

Other physical quantities as baryonic mass, angular momentum, polar red shift and circumferential radius show a similar behaviour: for fixed mass shed parameter or radius ratio they increase with increasing central pressure. Likewise they increase with decreasing mass shed parameter or decreasing 
Table 1. Properties of the maximal mass configuration. Only valid digits are given. An asterisk indicates that the corresponding quantity has a global maximum there.

\begin{tabular}{|c|c|c|}
\hline Physical quantity & value & \\
\hline Gravitational mass & $M=0.19435 \mu^{-1 / 2}$ & $*$ \\
\hline Baryonic mass & $M_{0}=0.27316 \mu^{-1 / 2}$ & * \\
\hline Angular velocity & $\Omega=1.8822 \mu^{1 / 2}$ & $*$ \\
\hline Angular momentum & $J=0.03637 \mu^{-1}$ & $*$ \\
\hline Polar radius & $r_{\mathrm{p}}=0.04856 \mu^{-1 / 2}$ & \\
\hline Equatorial radius & $r_{\mathrm{e}}=0.08475 \mu^{-1 / 2}$ & \\
\hline Radius ratio & $r_{\mathrm{p}} / r_{\mathrm{e}}=0.5730$ & \\
\hline Circumferential radius & $R_{\text {circ }}=0.41538 \mu^{-1 / 2}$ & * \\
\hline Polar red shift & $Z_{p}=7.378$ & $*$ \\
\hline
\end{tabular}

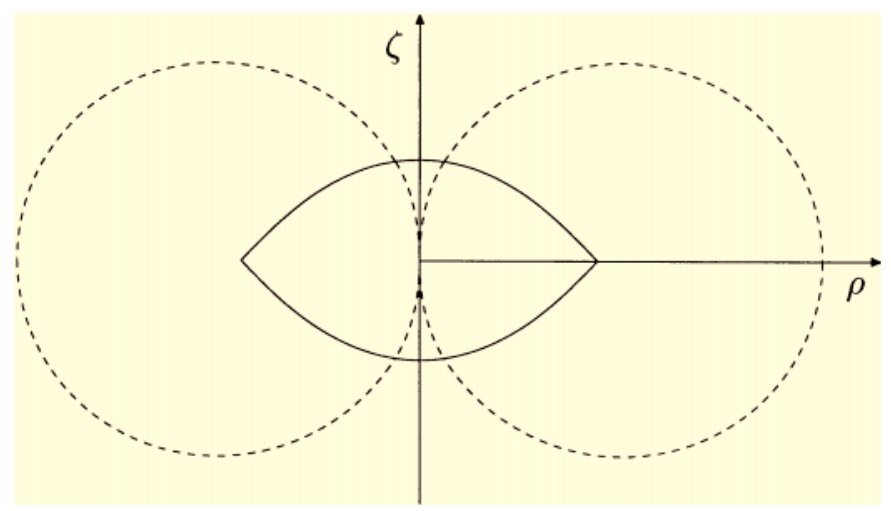

Fig. 4. Meridional coss section (solid line) and ergo-region (dashed line) of the maximal mass configuration. Axes are scaled identically.

radius ratio if the central pressure is kept constant. So a global maximum for each of them is obtained on the common edge of mass shed and infinite central pressure curves, corresponding thus to a very special limit star.

\section{Maximal mass configuration}

In Table 1 masses and other quantities are listed for this extreme configuration. Because it resides on two critical curves we notice a loss in accuracy that was somewhat recovered by an extrapolation to an infinite approximation order $m$ of the AKM method (cf. Ansorg et al. 2003c). Observe also the unexpectedly high value for the red shift $Z_{\mathrm{p}}$ of a photon emitted at one of the poles.

Figure 4 shows a meridional cross section of this configuration including the border of the ergo-region. As in general for diverging central pressure, the ergo-toroid degenerates by pinching together in the center.

We see the above work as a first step in the direction of the investigation of more realistic equations of state with the AKM method. The results, including the relation to relativistic ring solutions and further aspects like stability, will be published elsewhere.

Acknowledgements. We would like to thank Prof. R. Meinel and D. Petroff for valuable discussions and helpful advice. This work was supported by the Deutsche Forschungsgemeinschaft (DFG projects ME 1820/1-3 and SFB/TR 7 - B1).

\section{References}

Ansorg, M., Kleinwächter, A., \& Meinel, R. 2002, A\&A, 381, L49

Ansorg, M., Kleinwächter, A., \& Meinel, R. 2003a, ApJ, 582, L87

Ansorg, M., Kleinwächter, A., \& Meinel, R. 2003b, MNRAS, 339, 515

Ansorg, M., Kleinwächter, A., \& Meinel, R. 2003c, A\&A, 405, 711

Bardeen, J. M. 1971, ApJ, 167, 425

Bonazzola, S., Gourgoulhon, E., \& Marck, J. A. 1998, Phys. Rev. D, 58, 104020

Bonazzola, S., Gourgoulhon, E., Salgado, M., \& Marck, J. A. 1993, A\&A, 278, 421

Bonazzola, S., \& Schneider, S. 1974, ApJ, 191, 273

Butterworth, E. M., \& Ipser, J. R. 1975, ApJ, 200, L103

Butterworth, E. M., \& Ipser, J. R. 1976, ApJ, 204, 200

Chandrasekhar, S. 1967, ApJ, 147, 334

Dyson, F. W. 1892, Phil. Trans. Roy. Soc., 184, 43

Dyson, F. W. 1893, Phil. Trans. Roy. Soc., 184A, 1041

Eriguchi, Y., Hachisu, I., \& Nomoto, K. 1994, MNRAS, 266, 179

Eriguchi, Y., \& Sugimoto, D. 1981, Prog. Theor. Phys., 65, 1870

Friedman, J. L. 1998, in Black Holes and Relativistic Stars, ed. R. M. Wald (Chicago and London: The University of Chicago Press), 23

Friedman, J. L., Ipser, J. R., \& Parker, L. 1986, ApJ, 304, 115; Erratum 1990, ApJ, 351, 705

Friedman, J. L., Ipser, J. R., \& Parker, L. 1989, Phys. Rev. Lett., 62, 3015

Herold, H., \& Neugebauer, G. 1992, in Relativistic Gravity Research, Lecture Notes in Physics 410, ed. J. Ehlers, \& G. Schäfer (Berlin: Springer), 319

Komatsu, H., Eriguchi, Y., \& Hachisu, I. 1989a, MNRAS, 237, 355

Komatsu, H., Eriguchi, Y., \& Hachisu, I. 1989b, MNRAS, 239, 153

Kowalewsky, S. 1885, Astron. Nachr., 111, 37

Lattimer, J. M., Prakash, M., Masak, D., \& Yahil, A. 1990, ApJ, 355, 241

Neugebauer, G., \& Herold, H. 1992, in Relativistic Gravity Research, Lecture Notes in Physics 410, ed. J. Ehlers, \& G. Schäfer (Berlin: Springer), 305

Nozawa, T., Stergioulas, N., Gourgoulhon, E., \& Eriguchi, Y. 1998, A\&AS, 132, 431

Petroff, D. 2003, Ph. D. Thesis, university of Jena

Poincaré, H. 1885a, C. R. Acad. Sci., 100, 346

Poincaré, H. 1885b, Bull. Astr., 2, 109

Poincaré, H. 1885c, Bull. Astr., 2, 405

Stergioulas, N., \& Friedman, J. L. 1995, ApJ, 444, 306

Stergioulas, N. 1998, Living Rev. in Relativity, 1998-8, http://www. livingreviews.org

Wilson, J. R. 1972, ApJ, 176, 195

Wong, C. Y. 1974, ApJ, 190, 675 